\title{
A Discriminatory and Biorelevant Dissolution Test Method for Simvastatin Drug Products
}

\author{
N. Singla ${ }^{1}$, G. D. Gupta ${ }^{2}$, K. Kohli ${ }^{3}$, and A. K. Singla ${ }^{4}$ \\ 'Institute of Pharmacy, B.S.A.I.P, Faridabad, 129004 India \\ ${ }^{2}$ Amar Shaheed Baba Ajit Singh Jujhar Singh Memorial College of Pharmacy, Bela (Ropar), \\ 140111 India \\ ${ }^{3}$ Faculty of Pharmacy, Jamia Hamdard, New Delhi, 110062 India \\ ${ }^{4}$ Department of Pharmaceutical Research and Development, Ranbaxy Research Lab, Gurgaon, \\ 122001 India
}

\section{ABSTRACT}

Biorelevant in vitro dissolution is a useful technique for qualitative forecasting of the in vivo behavior of a formulation. A biorelevant dissolution medium for simvastatin was developed with a lower concentration of surfactant $(0.1 \%$ sodium lauryl sulfate, SLS) in the medium as compared with the $0.5 \%$ SLS concentration stated in the USP monograph. The slower dissolution rate of simvastatin tablets in $0.1 \%$ SLS relative to a self-emulsifying formulation of simvastatin correlated with the enhanced bioavailability of the self-emulsifying formulation in albino rats.

\section{INTRODUCTION}

T he dissolution test is utilized as either a research tool for optimizing new formulations or a quality control test to routinely monitor the uniformity and reproducibility of production batches. In biological systems, drug dissolution is an important attribute before systemic absorption (1). The dissolution test should reflect significant differences in bioavailability arising from differences in dissolution (2) and discriminate formulation factors such as polymers, particle surface area, or physical and chemical characteristics of the drug $(3,4)$. When dissolution testing is used to forecast the in vivo performance of a drug, it is critical that the in vitro test should mimic the in vivo conditions as closely as possible. The nature of the dissolution medium affects the dissolution rate. The preferred media are $\mathrm{pH} 4.5$ acetate/phosphate buffer, pH 6.8 phosphate buffer, $\mathrm{pH}$ 7.2/7.4 phosphate buffer, water, or $0.1 \mathrm{~N}$ hydrochloric acid (3). For poorly water-soluble drugs that do not show $\mathrm{pH}$-dependent solubility, an approach to increase the dissolution rate is the addition of wetting agents, solubilizing agents, or surfactants to the dissolution media (5). The use of surfactants in the dissolution media for sparingly soluble drugs is physiologically relevant and well-documented. Sodium lauryl sulfate is a surfactant commonly used in dissolution media for poorly soluble drugs (6). A dissolution medium containing surfactant can better simulate the environment of the gastrointestinal tract than a medium containing organic solvents or other nonphysiological substances. The addition of a small amount of surfactant below its critical micelle concentration is often sufficient to solubilize certain drug products (7). In those

${ }^{1}$ Corresponding author. cases where a higher concentration of surfactant leads to faster dissolution, any potential correlation with in vivo performance is lost; therefore, a low concentration of surfactant is a modifier of choice. The dissolution test is generally performed under sink conditions (i.e., the material already in solution should not exert a modifying effect on the rate of dissolution). Sink conditions occur in a volume of dissolution medium that is 3-10 times greater than the saturation volume for the drug. Drug dissolution or release-rate data based on a discriminating and well-designed test is of tremendous value in formulation development.

The USP monograph for dissolution testing of simvastatin tablets lists $\mathrm{pH} 7.0$ phosphate buffer with $0.5 \%$ SLS as the medium (8). SLS-containing Fasted State Simulated Gastric Fluid $\left(\mathrm{FaSSGF}_{\mathrm{SLS}}\right.$ ) is probably the most commonly used FaSSGF, having $0.25 \%$ (8.67 mM) SLS. A medium containing $0.5 \%(17.34 \mathrm{mM}) \mathrm{SLS}$ may result in solubility overestimation (9). Therefore, the objective of our study was to evaluate different concentration levels (0.1-0.5\%) of SLS in the dissolution medium for two solid oral dosage formulations of simvastatin and to determine any effect on the biorelevance of the dissolution method.

\section{EXPERIMENTAL \\ Materials}

Reagent grade disodium hydrogen phosphate and sodium hydroxide were obtained from S.D. Fine-Chem Ltd. Simvastatin was obtained from Ranbaxy Labs, India. Sodium lauryl sulfate and buffer ingredients were purchased from $\mathrm{CDH}$, India. Distilled water was used in the preparation of all test media. Simvastatin (Zocor) 40-mg tablets were obtained from Merck, USA. A self-microemulsifying formulation of simvastatin $40 \mathrm{mg}$ was manufactured in our lab; the formulation is described in Table 1. 
Table 1. Composition of Self-Emulsifying Formulation Filled in Capsules

\begin{tabular}{lcc}
\hline Ingredient & Weight $(\mathbf{m g})$ & \% w/w \\
\hline Simvastatin & 40.0 & 7.7 \\
\hline Labrasol & 362.0 & 69.6 \\
\hline Plurol oleique & 54.3 & 10.4 \\
\hline Transcutol & 6.0 & 1.2 \\
\hline Maisine oil & 57.6 & 11.1 \\
\hline
\end{tabular}

\section{Instrumentation}

A Lab India dissolution test apparatus equipped with a six-paddle assembly and a built-in system to regulate temperature and paddle rotation was employed. A double-beam UV spectrophotometer (Shimadzu, Japan) was used for analysis.

\section{Dissolution Method}

Dissolution testing of capsules filled with the self-emulsifying formulation and tablets (Zocor) was performed according to USP 32: Apparatus 2 (paddle) at $50 \mathrm{rpm} ; 37 \pm 0.5^{\circ} \mathrm{C} ; 900 \mathrm{~mL}$ of $\mathrm{pH} 7.0$ phosphate buffer $(0.01 \mathrm{M})$ with SLS in varying amounts from $0.1 \%$ to $0.5 \%$. The dissolution medium was prepared by dissolving 8.28 $\mathrm{g}$ of sodium dihydrogen phosphate $\left(\mathrm{NaH}_{2} \mathrm{PO}_{4} \cdot 2 \mathrm{H}_{2} \mathrm{O}\right)$ in $6 \mathrm{~L}$ of water and adjusting the $\mathrm{pH}$ to 7.0 with $\mathrm{NaOH}$. SLS was added in concentrations of $0.1-0.5 \% \mathrm{w} / \mathrm{v}$. Simvastatin release was quantified by UV spectrophotometric analysis of the dissolution samples at $239 \mathrm{~nm}$.

\section{Preparation of Reference Standard}

Simvastatin reference standard equivalent to $40 \mathrm{mg}$ was dissolved in a $100-\mathrm{mL}$ volumetric flask. Fifty milliliters of acetonitrile was added. The solution was sonicated to dissolve the drug and brought to volume with acetonitrile. The solution was diluted to $8 \mu \mathrm{g} / \mathrm{mL}$ with dissolution medium. Absorbance was measured at $239 \mathrm{~nm}$.

\section{In Vitro Dissolution Study}

The marketed formulation (tablet) containing $40 \mathrm{mg}$ simvastatin and the self-emulsifying formulation of simvastatin were evaluated using $0.1-0.5 \%$ SLS concentration. Ten milliliters of sample was withdrawn at intervals of $5,10,20$, and $30 \mathrm{~min}$.

\section{In Vivo Bioavailability Study}

Eighteen albino rats weighing between 150 and $200 \mathrm{~g}$ were used in the study. The study was conducted in an open, randomized, single-dose, crossover design under fasting conditions. Each animal received a dose equivalent to $1 \mathrm{mg}$ of simvastatin. Simvastatin formulations (40-mg tablets and self-emulsifying system, $40 \mathrm{mg}$ ) were suitably suspended in fixed volumes of purified water to

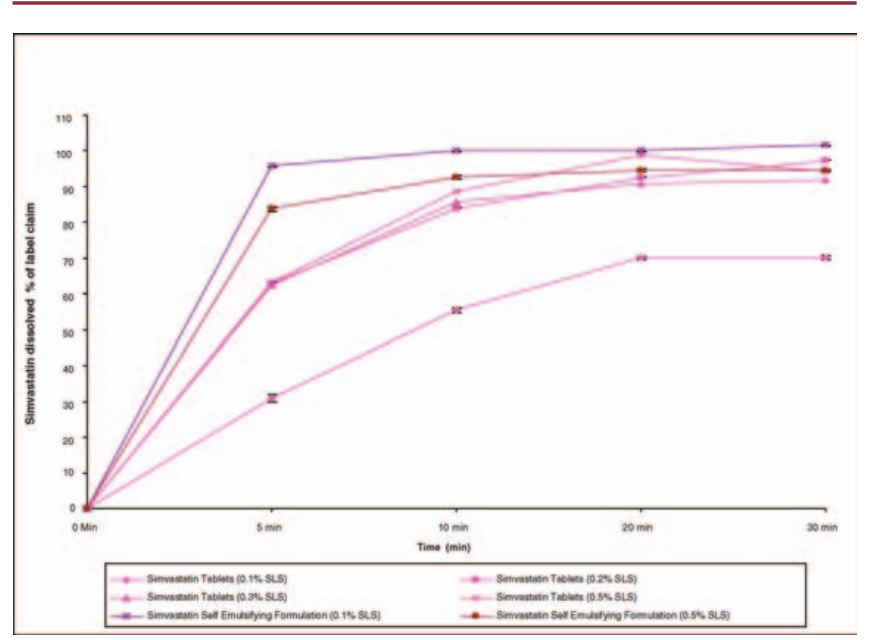

Figure 1. Dissolution profile of simvastatin drug products $(40 \mathrm{mg})$ in $\mathrm{pH} 7.0$ phosphate buffer with different concentrations of SLS.

provide a 1-mg/mL dose accurately in a cross-over design, observing a washout period of 7 days. Blood samples were collected at periods of $0.5,1,2,3,4,6,8,10$, and $12 \mathrm{~h}$ post dose. Plasma was separated and analyzed on an LC/MS/MS unit.

\section{RESULTS AND DISCUSSION}

The dissolution profiles for simvastatin tablets and self-emulsifying capsule formulation $(0.1,0.2,0.3$, and $0.5 \%$ SLS in the media) are shown in Figure 1. The dissolution profiles for both formulations are similar and essentially complete with SLS concentrations of $0.2-0.5 \%$ in the dissolution media. With $0.1 \% \mathrm{SLS}$, the dissolution of the tablets was only $70 \%$ at $30 \mathrm{~min}$, and there was no significant increase in dissolution with additional testing time. There was a marked improvement in the solubility of simvastatin in the self-emulsifying formulation as compared with the tablets. The self-emulsifying formulation showed rapid dissolution of simvastatin (complete release in $10 \mathrm{~min}$ ). This suggests that the simvastatin was released from the self-emulsifying formulation because of its small droplet size, which permits a faster release of drug into the aqueous phase, and this could affect the bioavailability. The in vitro dissolution was compared with the in vivo bioavailability for selecting a biorelevant medium. The various pharmacokinetic parameters are compared in Table 2. The self-emulsifying formulation showed significant improvement in bioavailability (1.5-fold) and faster onset compared with the tablets, as shown in Figure 2. Thus, the dissolution method using $0.1 \%$ SLS in the medium seems to be more biorelevant and discriminatory than methods utilizing higher concentrations of SLS.

\section{CONCLUSION}

The apparent intrinsic dissolution rate of poorly soluble drugs increased linearly with a surfactant medium due to 
Table 2. Pharmacokinetic Parameters of the Maximum Plasma Concentration $\left(C_{\max }\right)$ and Area Under the Curve $\left(A \cup C_{0-t}\right)$ for Simvastatin Following Oral Administration in Albino Rats

\begin{tabular}{lccc}
\hline $\begin{array}{l}\text { Pharmacokinetic } \\
\text { parameter }\end{array}$ & $\begin{array}{c}\text { Simvastatin } \\
\text { Self-Emulsifying } \\
\text { (Test) }\end{array}$ & $\begin{array}{c}\text { Simvastain } \\
\text { Tablets } \\
\text { (Reference) }\end{array}$ & $\begin{array}{c}\text { Relative } \\
\text { Bioavailability } \\
\text { (T/R ratio) }\end{array}$ \\
\hline$A \cup C_{0-t}(\mathrm{ng} \mathrm{h} / \mathrm{mL})$ & $47.68 \pm 13.75$ & $31.99 \pm 10.11$ & 1.49 \\
\hline$C_{\max }(\mathrm{ng} / \mathrm{mL})$ & $26.64 \pm 7.92$ & $15.59 \pm 5.16$ & - \\
\hline$T_{\max }(\mathrm{h})$ & $0.50 \pm 0.23$ & $1.00 \pm 0.43$ & - \\
\hline
\end{tabular}

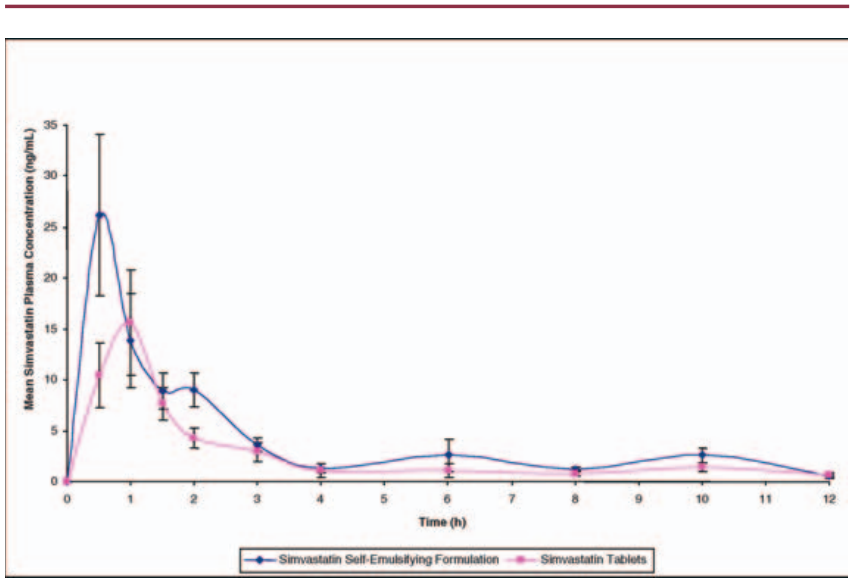

Figure 2. Linear plot of simvastatin plasma concentration after oral administration of simvastatin formulations in albino rats (1-mg dose).

an increase in wettability and solubility. For two formulations of poorly soluble simvastatin, a low concentration of surfactant $(0.1 \% \mathrm{SLS})$ in the dissolution medium was more biorelevant than was a higher concentration of surfactant. Creating sink conditions with higher surfactant concentrations may not be a proper approach if a biorelevant method is sought. The slower dissolution rate of simvastatin tablets in $0.1 \%$ SLS as compared with the self-emulsifying formulation of simvastatin was confirmed by comparison with in vivo results from bioavailability studies in albino rats.

\section{REFERENCES}

1. Dressman, J. B.; Amidon, G. L.; Reppas, C.; Shah, V. P. Dissolution testing as a prognostic tool for oral drug absorption: immediate release forms. Pharm. Res. 1998, 15 (1), 11-22.

2. Dissolution Testing of Solid Oral Dosage Forms. In British Pharmacopoeia; The Stationary Office: London, 2009; p A563.

3. The Dissolution Procedure: Development and Validation <1092>. In United States Pharmacopeia and National Formulary USP 32-NF 27; The United States Pharmacopeial Convention, Inc.: Rockville, MD, 2009; pp 599-604.
4. Hörter, D.; Dressman, J. B. Influence of physicochemical properties on dissolution of drugs in the gastrointestinal tract. Adv. Drug Deliver. Rev. 2001, $46,75-87$.

5. Shah, V. P.; Konecny, J. J.; Everett, R. L.; McCullough, B.; Noorizadeh, A. C.; Skelly, J. P. In vitro dissolution profile of water-insoluble drug dosage forms in the presence of surfactants. Pharm. Res. 1989, 6, 612-618.

6. Zhao, F.; Malayev, V.; Rao, V.; Hussain, M. Effect of SLS in dissolution media on dissolution of hard gelatin capsule shells. Pharm. Res. 2004, 21 (1), 144-148.

7. Noory, C.; Tran, N.; Ouderkirk, L.; Shah, V. Steps for development of a dissolution test for sparingly water-soluble drug products. Dissolution Technol. 2000, 7 (1), 16-21.

8. Aburub, A.; Risley, D. S.; Mishra, D. A critical evaluation of fasted state simulating gastric fluid (FaSSGF) that contain sodium lauryl sulfate and proposal of a modified recipe. Int. J. Pharm. 2007, 347, 16-22.

9. Simvaststin Tablets Monograph. In United States Pharmacopeia and National Formulary USP 32-NF 27; The United States Pharmacopeial Convention, Inc.: Rockville, MD, 2009; p 3558.

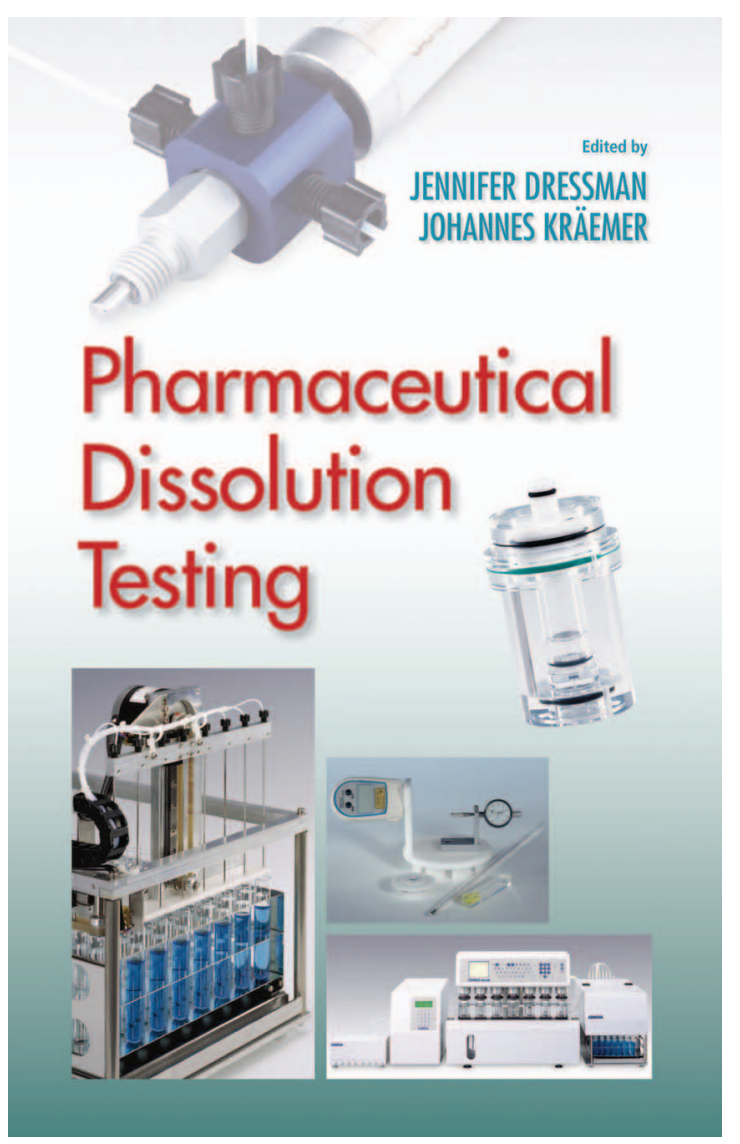

This book may be ordered online at the DT website at www.dissolutiontech.com. Price, including shipping, is $\$ 210.00$ for US and $\$ 230.00$ for outside USA. Credit card payment is available. 